\title{
Gleditsia sinensis thorn extract inhibits the proliferation and migration of PDGF-induced vascular smooth muscle cells
}

\author{
SUNG-SOO PARK ${ }^{1}$, WUN-JAE KIM ${ }^{2}$ and SUNG-KWON MOON ${ }^{3}$ \\ ${ }^{1}$ Department of Food Science and Nutrition, Jeju National University, Jeju, Jeju Special Self-Governing Province 690-756; \\ ${ }^{2}$ Department of Urology, Chungbuk National University College of Medicine, Cheongju, Chungbuk 361-763; \\ ${ }^{3}$ School of Food Science and Technology, Chung-Ang University, Anseong, Gyeonggi-Do 456-756, Republic of Korea
}

Received November 18, 2013; Accepted March 19, 2014

DOI: $10.3892 / \mathrm{mmr} .2014 .2422$

\begin{abstract}
The thorns of Gleditsia sinensis have been used to prevent or treat numerous diseases. The present study aimed to investigate the molecular mechanism of the ethanol extract of Gleditsia sinensis thorns (EEGS) on platelet-derived growth factor (PDGF)-treated vascular smooth muscle cells (VSMCs). EEGS treatment was found to inhibit DNA synthesis in PDGIF-treated VSMCs in a dose-dependent manner, without cell toxicity. These inhibitory effects were associated with $\mathrm{G}_{1}$-phase cell-cycle arrest, which was caused by the decreased expression of cyclins and cyclin-dependent kinases (CDKs) and the upregulation of p27KIP1 expression in PDGF-stimulated VSMCs. Among the pathways examined, EEGS treatment was observed to only inhibit the PDGF-induced phosphorylation of Akt. In addition, EEGS treatment suppressed the migration and invasion of VSMCs in the presence of PDGF as determined by wound-healing and Matrigel ${ }^{\mathrm{TM}}$ invasion assays. Furthermore, zymographic and western blot analyses revealed that EEGS treatment suppressed matrix metalloproteinase (MMP)-9 expression in PDGF-treated VSMCs, which was attributed to a reduction in the binding activities of nuclear factor $\kappa$-light-chain-enhancer of activated $B$ cells $(N F-\kappa B)$, activator protein (AP)-1 and specificity protein (Sp)-1. These results demonstrate that EEGS induces p27KIP1-mediated $\mathrm{G}_{1}$-phase cell-cycle arrest, reduces Akt phosphorylation and prevents MMP-9 expression by decreasing the binding activities of NF- $\mathrm{B}$, AP-1 and Sp-1 in PDGF-treated VSMCs, thus resulting in growth inhibition and the suppression of migration and invasion. These results may suggest a novel perspective for the use of EEGS in the treatment and prevention of vascular proliferative diseases.
\end{abstract}

Correspondence to: Dr Sung-Kwon Moon, School of Food Science and Technology, Chung-Ang University, 4726 Seodong-daero, Daedeok-Myeon, Anseong, Gyeonggi-Do 456-756, Republic of Korea

E-mail: sumoon66@dreamwiz.com

Key words: ethanol extract of Gleditsia sinensis thorns, VSMCs, PDGF, $\mathrm{G}_{1}$-phase cell cycle arrest, p27KIP1, migration, invasion

\section{Introduction}

The proliferation and migration of vascular smooth muscle cells (VSMCs), which is associated with neointimal formation, is a critical pathological process in the formation of vascular lesions and may result in atherosclerosis and restenosis $(1,2)$. Growth factors and cytokines produced by several types of cells, including macrophages, endothelial cells and VSMCs, contribute to the development and progression of vascular lesions (3). Platelet-derived growth factor (PDGF), a significant regulator of mitogenesis, influences the proliferation of VSMCs through the activation of extracellular signal-regulated kinase (ERK) 1/2, c-Jun N-terminal kinase (JNK), p38 mitogen-activated protein kinase (MAPK) and Akt signaling pathways $(4,5)$.

VSMCs proliferate and migrate into the intimal layer following vascular injury in atherosclerotic lesions (1). The proliferation of VSMCs is primarily controlled by regulation of the cell cycle, which is composed of four distinct sequential phases, known as $G_{0} / G_{1}, S, G_{2}$ and $M(6)$. Following proliferative stimulation, VSMCs enter $S$ phase from the quiescent $\mathrm{G}_{0} / \mathrm{G}_{1}$ phase (6). This $\mathrm{G}_{1}$ - to $\mathrm{S}$-phase transition is regulated by cyclin/cyclin-dependent kinase (CDK) complexes, including cyclin D1/CDK4 and cyclin E/CDK2, which are inhibited by the negative regulators p21WAF1 and p27KIP1 $(7,8)$.

Matrix metalloproteinases (MMPs), including the gelatinases MMP-2 and MMP-9, degrade type IV collagen, which leads to the migration and invasion of VSMCs, resulting in the intimal thickening that is characteristic of vascular plaque instability (9). MMP expression is tightly regulated at the transcriptional level by growth factors, cytokines, hormones and tumor promoters $(1,9)$. In vitro and in vivo studies have shown that MMP-9 expression is critical in the progression of arterial lesions (9-13). In VSMCs, MMP-9 expression is regulated by various stimuli, including growth factors and cytokines secreted by platelets, macrophages and VSMCs (9-15).

The thorns of Gleditsia sinensis are used in traditional medicinal treatments and have been shown to exert anti-cancer, anti-mutagenic, anti-allergenic, anti-microbial, anti-human immunodeficiency virus and anti-inflammatory effects (16-21). The primary components of Gleditsia sinensis are stigmasterol (16), ellagic acid glycosides (22), flavonoids (17) and lupane acid (18). We have previously demonstrated that the ethanol extract of Gleditsia sinensis thorns (EEGS) is capable 
of inhibiting tumor necrosis factor $\alpha$-induced VSMC proliferation (23). However, the molecular mechanism underlying the inhibitory effect of EEGS in the proliferation and migration of PDGF-induced VSMCs is yet to be elucidated. Therefore, the present study aimed to investigate the inhibitory effects of EEGS on cell proliferation and migration in PDGF-induced VSMCs.

\section{Materials and methods}

Materials. Polyclonal antibodies against cyclin E, CDK2 and CDK4 were obtained from Santa Cruz Biotechnology, Inc. (Santa Cruz, CA, USA). Polyclonal antibodies against cyclin D1, p21WAF1, p53, p27, ERK, phosphorylated (phospho)-ERK, p38 MAPK, phospho-p38 MAPK, JNK, phospho-JNK, Akt, phospho-Akt and GAPDH were obtained from New England Biolabs Inc. (Ipswich, MA, USA). The polyclonal MMP-9 antibody was obtained from Chemicon (Temecula, CA, USA). All experimental procedures were and protocols were approved by the Ethics Committee of Chung-Ang University (Anseong, Republic of Korea).

Preparation of extract. A total of $100 \mathrm{~g}$ air-dried Gleditsia sinensis thorns were crushed, prior to the addition of ethanol. Extraction was performed by heating to $100^{\circ} \mathrm{C}$. The extract was then concentrated using a rotary evaporator, and lyophilized. The final extract, which weighed $10 \mathrm{~g}$ (a collection rate of $10 \%$ ), was diluted in saline solution.

Cell cultures. Aortic smooth muscle cells were obtained from the aortas of young male Sprague Dawley rat (8 weeks old, 200-250 g) using enzymatic digestion, as described previously (23). VSMCs were isolated from the Sprague Dawley rats. Briefly, the aortas were removed under sterile conditions. Following being rinsed several times in Hanks' balanced salt solution, the adventitia was removed from the aortas and the aortas were homogenized and digested using $5 \mathrm{ml}$ digestion solution $(0.125 \mathrm{mg} / \mathrm{ml}$ elastase, $0.25 \mathrm{mg} / \mathrm{ml}$ soybean trypsin inhibitor, $10 \mathrm{mg} / \mathrm{ml}$ collagenase I, $2.0 \mathrm{mg} / \mathrm{ml}$ crystallized bovine albumin and $15 \mathrm{mM}$ HEPES) at $37^{\circ} \mathrm{C}$ for $45 \mathrm{~min}$. The cellular digests were filtered through a sterile $100-\mu \mathrm{m}$ nylon mesh, centrifuged at $184 \mathrm{x} \mathrm{g}$ for $10 \mathrm{~min}$ and washed twice in Dulbecco's modified Eagle's medium (DMEM) containing 10\% fetal calf serum, prior to being cultured in the same medium. Experiments shown are representative of the results from three independent cultures from each group of rats. VSMC characterization was performed by immunofluorescence staining using a monoclonal antibody against smooth muscle- $\alpha$-actin (Sigma Aldrich, St. Louis, MO, USA). These explants were incubated in DMEM containing 10\% fetal bovine serum (FBS), $2 \mathrm{mM}$ glutamine, $50 \mu \mathrm{g} / \mathrm{ml}$ gentamycin and $50 \mu \mathrm{l} / \mathrm{ml}$ amphotericin-B at $37^{\circ} \mathrm{C}$ in a humidified $5 \% \mathrm{CO}_{2}$ atmosphere. Cells were passaged every 3-5 days and experiments were performed on cells at primary culture passages five to eight. In the majority of experiments, cells at a confluence of $80-90 \%$ were made quiescent by $24 \mathrm{~h}$ of incubation in DMEM without FBS.

Cell viability assay. Growth-arrested VSMCs were incubated on 24-well plates with EEGS for varying time-periods in the presence of PDGF. Cell viability was determined using a modified MTT assay, which was based on the conversion of tetrazolium salt 3-(4,5-dimethylthiazol-2-yl)-5-(3-carboxym-thoxyphenyl)-2-(4-sulfophenyl)-2-tetrazolium to the formazan product by mitochondrial dehydrogenases (23). Formazan was quantified by measuring the absorbance at $490 \mathrm{~nm}$.

$\left[{ }^{3} H\right]$ thymidine incorporation. VSMCs, grown to near-confluence in 24-well tissue culture plates, were made quiescent and treated with EEGS in the presence of PDGF, as indicated. The $\left[{ }^{3} \mathrm{H}\right]$ thymidine incorporation experiment was performed as described previously (23).

Cell-cycle analysis using fluorescence-activated cell sorting (FACS). Cells were harvested, fixed in $70 \%$ ethanol and stored at $-20^{\circ} \mathrm{C}$. Cells were then washed twice with ice-cold phosphate-buffered saline (PBS) and incubated with RNase, the DNA intercalating dye and propidium iodide. Cell-cycle phase analysis was performed using a Becton Dickinson FACStar $^{\text {TM }}$ flow cytometer equipped with Becton Dickinson Cell Fit software (BD Biosciences, Franklin Lakes, NJ, USA).

Western blot analysis. Quiescence was induced in VSMCs, which were grown to near-confluence in 100-mm tissue culture plates, prior to treatment with EEGS in the presence of PDGF for varying durations at $37^{\circ} \mathrm{C}$. Cells were then washed twice with cold PBS and freeze-thawed in $250 \mu 1$ lysis buffer [50 mmol/1 HEPES ( $\mathrm{pH} 7.5), 150 \mathrm{mmol} / \mathrm{l} \mathrm{NaCl}, 1 \mathrm{mmol} / 1$ EDTA, $2.5 \mathrm{mmol} / 1$ ethylene glycol tetraacetic acid (EGTA), $1 \mathrm{mmol} / 1$ dithiothreitol (DTT), $10 \mathrm{mmol} / 1 \beta$-glycerophosphate, $1 \mathrm{mmol} / 1 \mathrm{NaF}, 0.1 \mathrm{mmol} / 1 \mathrm{Na}_{3} \mathrm{VO}_{4}, 0.1 \mathrm{mmol} / 1$ phenylmethylsulfonyl fluoride (PMSF), 10\% glycerol, 0.1\% Tween-20, $10 \mu \mathrm{g} / \mathrm{ml}$ leupeptin and $2 \mu \mathrm{g} / \mathrm{ml}$ aprotinin]. Cell lysates were then harvested into $1.5-\mathrm{ml}$ tubes and placed on ice for $15 \mathrm{~min}$, prior to centrifugation at $10,786 \mathrm{x} \mathrm{g}$ for $20 \mathrm{~min}$ at $4^{\circ} \mathrm{C}$. The protein concentration of the supernatant was determined using the Bradford Protein Assay (Bio-Rad, Hercules, CA, USA). Equal quantities of cellular proteins were resolved by electrophoresis on a $0.1 \%$ SDS- $10 \%$ polyacrylamide gel under denaturing conditions. The proteins were then electrophoretically transferred to nitrocellulose membranes (Amersham Biosciences Corp., Piscataway, NJ, USA). Following blocking in $10 \mathrm{mmol} / \mathrm{l}$ Tris- $\mathrm{HCl}(\mathrm{pH} 8.0), 150 \mathrm{mmol} / \mathrm{l} \mathrm{NaCl}$ and $5 \%$ (w/v) non-fat dry milk, membranes were incubated with primary antibodies for $90 \mathrm{~min}$ and then further incubated with peroxidase-conjugated secondary antibodies for $45 \mathrm{~min}$. Immunocomplexes were detected using a chemiluminescence reagent kit (Amersham Biosciences Corp.). The experiments were repeated at least three times (13).

Immunoprecipitation and immune-complex kinase assays. Cell lysates were prepared using ice-cold lysis buffer [50 $\mathrm{mmol} / 1$ HEPES (pH 6.0), 150 mmol/1 NaCl, 1 mmol/1 EDTA, 2.5 mmol/1 EGTA, $1 \mathrm{mmol} / 1 \mathrm{DTT}, 10 \mathrm{mmol} / 1 \beta$-glycerophosphate, $1 \mathrm{mmol} / 1$ $\mathrm{NaF}, 0.1 \mathrm{mmol} / 1 \mathrm{Na}_{3} \mathrm{VO}_{4}, 0.1 \mathrm{mmol} / 1 \mathrm{PMSF}, 10 \%$ glycerol, $0.1 \%$ Tween-20, $10 \mu \mathrm{g} / \mathrm{ml}$ leupeptin and $2 \mu \mathrm{g} / \mathrm{ml}$ aprotinin] and sonicated twice for $10 \mathrm{sec}$ using a Micro Ultrasonic Cell Disrupter (Kontes; Kimble Chase, LLC, Vineland, NJ, USA) at $30 \%$ power and $4^{\circ} \mathrm{C}$. Lysates were clarified by centrifugation at $10,000 \mathrm{xg}$ for $5 \mathrm{~min}$, and the supernatants were precipitated 
by treatment with protein A-Sepharose beads precoated with saturating quantities of the indicated antibodies at $4^{\circ} \mathrm{C}$ for $2 \mathrm{~h}$. When monoclonal antibodies were used, protein A-Sepharose was pretreated with rabbit anti-mouse immunoglobulin $G$ (Jackson ImmunoResearch Laboratories, Inc., West Grove, MA, USA). Proteins that were immunoprecipitated on the beads were washed four times with $1 \mathrm{ml}$ lysis buffer and twice with kinase buffer $\left(50 \mathrm{mmol} / 1 \mathrm{HEPES}, 10 \mathrm{mmol} / 1 \mathrm{MgCl}_{2}\right.$, $1 \mathrm{mmol} / \mathrm{l} \mathrm{DTT}, 10 \mathrm{mmol} / \mathrm{l} \beta$-glycerophosphate, $1 \mathrm{mmol} / \mathrm{l}$ $\mathrm{NaF}$ and $0.1 \mathrm{mmol} / 1$ sodium orthovanadate). The final pellet was resuspended in $25 \mu \mathrm{l}$ kinase buffer containing either $1 \mu \mathrm{g}$ glutathione $S$-transferase (GST)-retinoblastoma protein $(\mathrm{pRb})$ C-terminal (pRb amino acids 769 to 921) fusion protein (Santa Cruz Biotechnology, Inc.) or $5 \mu \mathrm{g}$ histone $\mathrm{H}_{1}$ (Invitrogen Life Technologies, Carlsbad, CA, USA), $20 \mu \mathrm{mol} / 1$ ATP and $5 \mu \mathrm{Ci}$ $\left[\gamma^{32} \mathrm{P}\right]$ ATP $(4,500 \mu \mathrm{Ci} / \mathrm{mmol}$; ICN Pharmaceuticals Inc., Costa Mesa, CA, USA), and incubated for $20 \mathrm{~min}$ at $30^{\circ} \mathrm{C}$ with occasional mixing. The reaction was terminated by the addition of $25 \mu \mathrm{l} 2 \mathrm{X}$ Laemmli sample buffer and separated on 10 or $12.5 \%$ SDS-polyacrylamide gels. The migration of histone $\mathrm{H}_{1}$ and GST-pRb was determined by Coomassie blue staining, and phosphorylated $\mathrm{pRb}$ and histone $\mathrm{H}_{1}$ were visualized.

Wound-healing migration assay. The growth-arrested cells were damaged using a 2-mm-wide tip. Cells were then treated with EEGS either alone or with PDGF. Cells were subsequently allowed to migrate and images were captured using an inverted microscope (magnification, $\mathrm{x} 40$ ).

Invasion assay. The growth-arrested cells were resuspended with EEGS either alone or with PDGF, in $100 \mu \mathrm{l}$ medium. Cells were placed in the upper section of the Transwell plate and incubated for $24 \mathrm{~h}$. Cells had to pass through a polycarbonate membrane that had a thin layer of extracellular matrix (ECM)-like material with $8-\mu \mathrm{m}$ pores. The ability of the cells to invade the ECM-like material was determined using a commercial cell invasion assay kit (Chemicon), as described previously.

Zymography. The conditioned medium was electrophoresed in a polyacrylamide gel containing $1 \mathrm{mg} / \mathrm{ml}$ gelatin. The gel was then washed at room temperature for $2 \mathrm{~h}$ with $2.5 \%$ Triton X-100 and maintained at $37^{\circ} \mathrm{C}$ overnight in a buffer containing $10 \mathrm{mM} \mathrm{CaCl}_{2}, 150 \mathrm{mM} \mathrm{NaCl}$ and $50 \mathrm{mM}$ Tris- $\mathrm{HCl}$ $(\mathrm{pH} 7.5)$. The gel was stained with $0.2 \%$ Coomassie blue and images were obtained using a light box. Proteolysis was detected as a white zone in a dark blue field.

Nuclear extracts and electrophoretic mobility shift assay (EMSA). Nuclear extracts were prepared as described previously (13). Cultured cells were collected by centrifugation and washed and suspended in a buffer containing $10 \mathrm{mM}$ HEPES (pH 7.9), 10 mM KCl, 0.1 mM EDTA, 0.1 mM EGTA, 1 mM DTT and 0.5 mM PMSF. After $15 \mathrm{~min}$ on ice, cells were vortexed in the presence of $0.5 \%$ Nonidet P-40. The nuclear pellet was then collected by centrifugation and extracted in a buffer containing $20 \mathrm{mM}$ HEPES ( $\mathrm{pH}$ 7.9), $0.4 \mathrm{M} \mathrm{NaCl}, 1 \mathrm{mM}$ EDTA, $1 \mathrm{mM}$ EGTA, $1 \mathrm{mM}$ DTT and $1 \mathrm{mM}$ PMSF for $15 \mathrm{~min}$ at $4^{\circ} \mathrm{C}$.

The nuclear extract $(10-20 \mu \mathrm{g})$ was preincubated at $4^{\circ} \mathrm{C}$ for 30 min with a 100 -fold excess of an unlabeled oligonucleotide spanning the -79 MMP-9 cis element of interest. The sequences were as follows: AP-1, CTGACCCCTGAGTCAGCACTT; Sp-1, GCCCATTCCTTCCGCCCCCAGATGAAGCAG and NF- $\kappa$ B, CAGTGGAATTCCCCAGCC. The reaction was then incubated at $4^{\circ} \mathrm{C}$ for $20 \mathrm{~min}$ in a buffer containing $25 \mathrm{mM}$ HEPES (pH 7.9), $0.5 \mathrm{mM}$ EDTA, $0.5 \mathrm{mM}$ DTT, $0.05 \mathrm{M}$ $\mathrm{NaCl}$ and $2.5 \%$ glycerol, with $2 \mu \mathrm{g}$ poly(deoxyinosinic-deoxycytidylic) acid and $5 \mathrm{fmol}\left(2 \times 10^{4} \mathrm{cpm}\right)$ Klenow end-labeled ( ${ }^{32} \mathrm{P}$-ATP) 30-mer oligonucleotide, which spanned the DNA binding site in the MMP-9 promoter. The reaction mixture was electrophoresed at $4{ }^{\circ} \mathrm{C}$ on a $6 \%$ polyacrylamide gel using a Tris-borate-EDTA ( $89 \mathrm{mM}$ Tris, $89 \mathrm{mM}$ boric acid and $1 \mathrm{mM}$ EDTA) running buffer. The gel was rinsed with water, dried and exposed to X-ray film overnight.

Statistical analysis. Where appropriate, data are expressed as the mean \pm standard error of the mean. Data were analyzed using factorial analysis of variance and a Fisher's least significant difference test, where appropriate. A value of $\mathrm{P}<0.05$ was considered to indicate a statistically significant difference.

\section{Results}

EEGS inhibits PDGF-stimulated VSMC proliferation. An MTT assay was used to assess the effect of EEGS on cell viability. PDGF treatment for $24 \mathrm{~h}$ was observed to increase VSMC viability approximately two-fold compared with the non-treated control VSMCs (Fig. 1A). However, treatment with EEGS $(100 \mu \mathrm{g} / \mathrm{ml})$ was found to inhibit the increase in cell viability induced by PDGF to a controlled level (Fig. 1A). EEGS treatment either alone $(12.5-100 \mu \mathrm{g} / \mathrm{ml})$ or with a vehicle (ethanol) was not observed to affect cell viability (Fig. 1A). To examine whether EEGS treatment inhibited PDGF-induced cell proliferation, a thymidine uptake incorporation assay was used. VSMCs were pretreated with $12.5,25,50$ or $100 \mu \mathrm{g} / \mathrm{ml}$ EEGS for $40 \mathrm{~min}$, then stimulated with PDGF (20 ng/ml) for $24 \mathrm{~h}$. PDGF-treated cells showed a significant increase in thymidine uptake compared with the control cells $(\mathrm{P}<0.05$; Fig. 1B). This PDGF-induced cell proliferation was significantly inhibited by EEGS in a concentration-dependent manner $(\mathrm{P}<0.05$; Fig. 1B).

EEGS induces $G_{1}$-phase cell-cycle arrest in PDGF-stimulated VSMC proliferation. Flow cytometric analysis was used to assess whether the anti-proliferative effect of EEGS was due to cell-cycle arrest in a specific phase. PDGF treatment was observed to significantly increase the proportion of VSMCs in the $S$ and $G_{2} / M$ phases of the cell cycle, with a concomitant decrease in the proportion in $\mathrm{G}_{1}$ phase, compared with the control cells $(\mathrm{P}<0.05$; Table I). However, EEGS $(100 \mu \mathrm{g} / \mathrm{ml})$ treatment in the presence of PDGF was observed to markedly reduce the percentage of cells in the $S$ and $G_{2} / M$ phases, resulting in a significant accumulation of cells in $\mathrm{G}_{1}$ phase, compared with the PDGF-treated VSMCs ( $\mathrm{P}<0.05$; Table I). These data indicate that EEGS treatment had an inhibitory effect on PDGF-induced VSMC proliferation, through the suppression of $G_{1}$ - to $S$-phase transition.

EEGS-induced $G_{1}$-phase cell-cycle arrest is associated with a decrease in cyclin-related kinase activity. To investigate the mechanism of EEGS-induced $\mathrm{G}_{1}$-phase cell-cycle arrest, 
Table I. Flow cytometric analysis of quiescent vascular smooth muscle cells treated with PDGF and various concentrations of EEGS.

\begin{tabular}{lccc}
\hline Group & $\mathrm{G}_{0} / \mathrm{G}_{1}$ phase $(\%)$ & $\mathrm{S}$ phase $(\%)$ & $\mathrm{G}_{2} / \mathrm{M}$ phase $(\%)$ \\
\hline Control & 75.51 & 16.52 & 7.97 \\
PDGF $(20 \mathrm{ng} / \mathrm{ml})$ & 53.24 & 30.79 & 15.97 \\
PDGF + EEGS $(25 \mu \mathrm{g} / \mathrm{ml})$ & 56.86 & 28.64 & 14.50 \\
PDGF + EEGS $(50 \mu \mathrm{g} / \mathrm{ml})$ & 68.68 & 17.52 & 13.80 \\
PDGF + EEGS $(100 \mu \mathrm{g} / \mathrm{ml})$ & 66.74 & 18.52 & 14.74
\end{tabular}

Values are presented as the mean from three experiments. PDGF, platelet-derived growth factor; EEGS, ethanol extract of Gleditsia sinensis thorns.
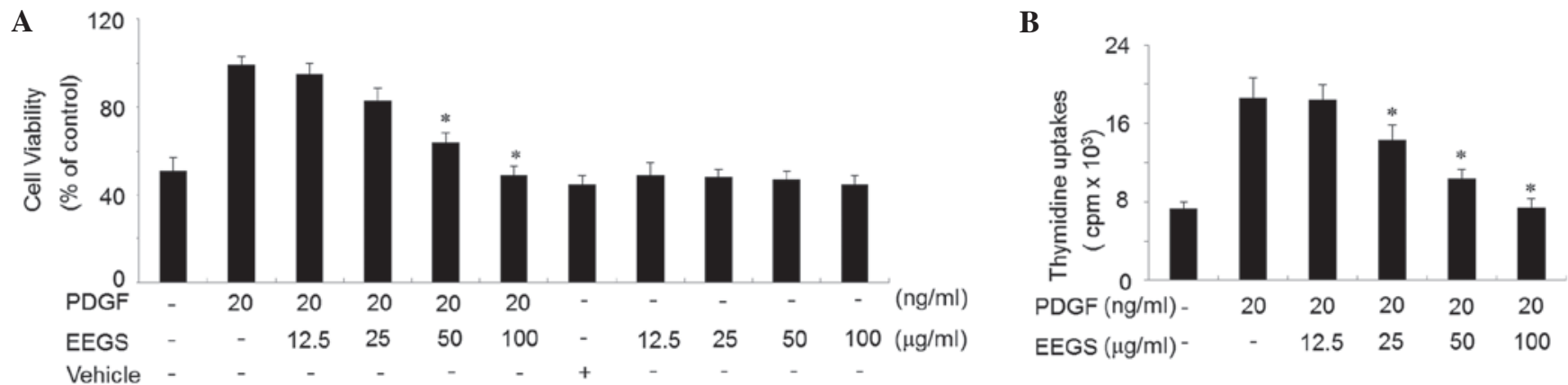

Figure 1. EEGS inhibits cell proliferation and induces $\mathrm{G}_{1}$-phase cell cycle arrest in PDGF-treated VSMCs. (A) VSMCs were incubated in serum-free medium for 1 day to induce quiescence, PDGF $(20 \mathrm{ng} / \mathrm{ml})$ or PDGF plus indicated concentrations of EEGS was added and the cells were cultured for a further $24 \mathrm{~h}$. Cell viability was assessed using a modified MTT assay. Results are presented as the mean \pm SE from three experiments. ${ }^{*}<0.05$ compared with PDGF treatment alone. (B) Measurement of DNA synthesis by thymidine uptake as a marker of cell proliferation. Quiescent VSMCs were treated with PDGF (20 ng/ml) in the presence or absence of EEGS for $24 \mathrm{~h}$. The cells were labeled with [methyl- $\left.{ }^{3} \mathrm{H}\right]$ thymidine at $1 \mu \mathrm{Ci} / \mathrm{ml}$ for the final $12 \mathrm{~h}$ of the culture period. Results are presented as the mean \pm SE from three experiments. ${ }^{*} \mathrm{P}<0.05$ compared with PDGF treatment alone. PDGF, platelet-derived growth factor; EEGS, ethanol extract of Gleditsia sinensis thorns; VSMC, vascular smooth muscle cell; SE, standard error.

the effects of EEGS on cyclins and CDKs were examined. As shown in Fig. 2A, PDGF treatment was observed to increase the expression of cyclin D1, cyclin E, CDK2 and CDK4 in VSMCs, and these effects were significantly inhibited with the addition of EEGS. The kinase activities of CDKs control cell-cycle transition (6-8). Therefore, the kinase activities associated with CDK2 and CDK4 were examined in EEGS-treated cells in the presence of PDGF. Treatment of VSMCs with PDGF was observed to significantly increase the kinase activities of the CDK2- and CDK4-immunoprecipitates (Fig. 2B). Furthermore, the PDGF-induced CDK2 and CDK4 activities were inhibited by EEGS treatment (Fig. 2B).

p27KIPl expression is associated with EEGS-induced $G_{1}$-phase cell-cycle arrest. $\mathrm{G}_{1}$ - to $\mathrm{S}$-phase cell-cycle progression is negatively regulated by cyclin-dependent kinase inhibitors (CDKIs), including p27KIP1 (7,8). Therefore, the effect of EEGS on p27KIP1 expression was assessed in PDGF-treated VSMCs. In the serum-starved quiescent VSMCs, p27KIP1 was constitutively expressed, and PDGF was observed to suppress its expression (Fig. 2C). Pretreatment with EEGS was found to reverse the PDGF-induced downregulation of p27KIP1 (Fig. 2C). By contrast, p21WAF1 expression increased with the addition of PDGF and remained unchanged with the addition of EEGS (Fig. 2C). Furthermore, PDGF reduced the protein expression of p53, and this was not affected by EEGS addition (Fig. 2C). It is well established that a reduction in kinase activity is involved in the increased interaction between p27KIP1 and CDKs $(7,8)$. Therefore, the effects of EEGS on p27KIP1-CDK interactions were assessed using an immunoprecipitation assay. The interaction between CDK2 and p27KIP1 was observed to be downregulated in PDGF-treated VSMCs (Fig. 2D). This interaction was upregulated in the presence of EEGS (Fig. 2D). Under similar experimental conditions, levels of the p27KIP1-CDK4 complexes were also increased in EEGS-treated cells following PDGF stimulation (Fig. 2D). These results indicate that p27KIP1 may be involved in EEGS-induced $\mathrm{G}_{1}$-phase cell-cycle arrest in PDGF-treated VSMCs.

EEGS inhibits PDGF-stimulated Akt phosphorylation in VSMCs. The effect of PDGF treatment on the phosphorylation of MAPK and Akt in VSMCs was next investigated. After 10 min PDGF treatment, an increase in the phosphorylation of ERK1/2, JNK, p38MAPK and Akt was observed, with no effect on the total protein levels of these molecules (Fig. 3). The inhibitory effect of EEGS on PDGF-induced ERK1/2, JNK, p38MAPK and Akt phosphorylation was also investigated. Pretreatment of VSMCs with EEGS was found to significantly inhibit Akt phosphorylation in PDGF-treated VSMCs (Fig. 3). However, PDGF-induced phosphorylation of ERK1/2, JNK 
A

$$
\begin{array}{ccccccc}
\text { PDGF } & - & + & + & + & + & (20 \mathrm{ng} / \mathrm{ml}) \\
\text { EEGS } & - & - & 25 & 50 & 100 & (\mu \mathrm{g} / \mathrm{ml})
\end{array}
$$

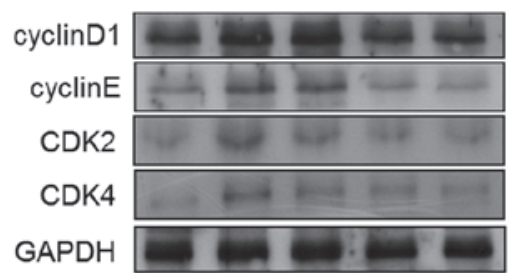

C

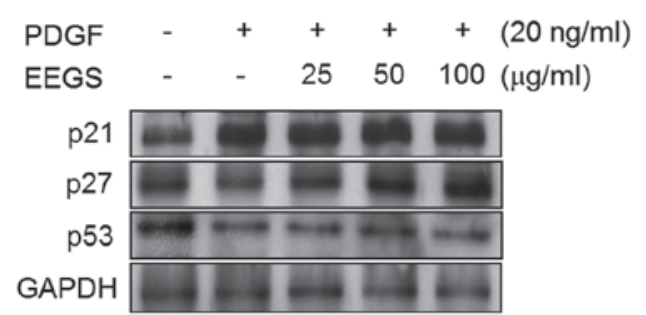

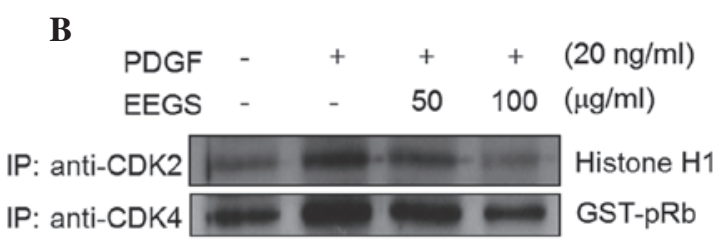

D

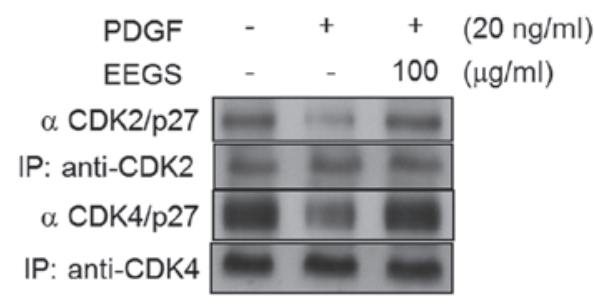

Figure 2. EEGS induces $\mathrm{G}_{1}$-phase cell cycle arrest through the expression of p27KIP1 in PDGF-treated VSMCs. Quiescent VSMCs were stimulated with PDGF (20 ng/ml) in the presence or absence of the indicated concentrations of EEGS for $24 \mathrm{~h}$. (A and C) Western blot analysis was performed using antibodies specific for cyclin D1, cyclin E, CDK2, CDK4, p21WAF1, p27KIP1 and p53. The results were normalized to GAPDH expression. (B) Total cell lysates were immunoprecipitated using anti-CDK2 and -CDK4 antibodies. The kinase reaction was performed using histone $\mathrm{H}_{1}$ (for $\mathrm{CDK}_{2}$ ) or $\mathrm{GST}_{\mathrm{-Rb}}$ (for $\mathrm{CDK}_{4}$ ) as substrates. (D) Equal quantities of cell lysates were subjected to immunoprecipitation using anti-CDK2 and -CDK4 antibodies, followed by SDS-PAGE. Following electrophoresis, samples were transferred to nitrocellulose membranes, and western blot analysis was performed using an anti-p27KIP1 antibody. PDGF, platelet-derived growth factor; EEGS, ethanol extract of Gleditsia sinensis thorns; VSMC, vascular smooth muscle cell; CDK, cyclin-dependent kinase; GST, glutathione $S$-transferase; pRb, retinoblastoma protein; IP, immunoprecipitation.

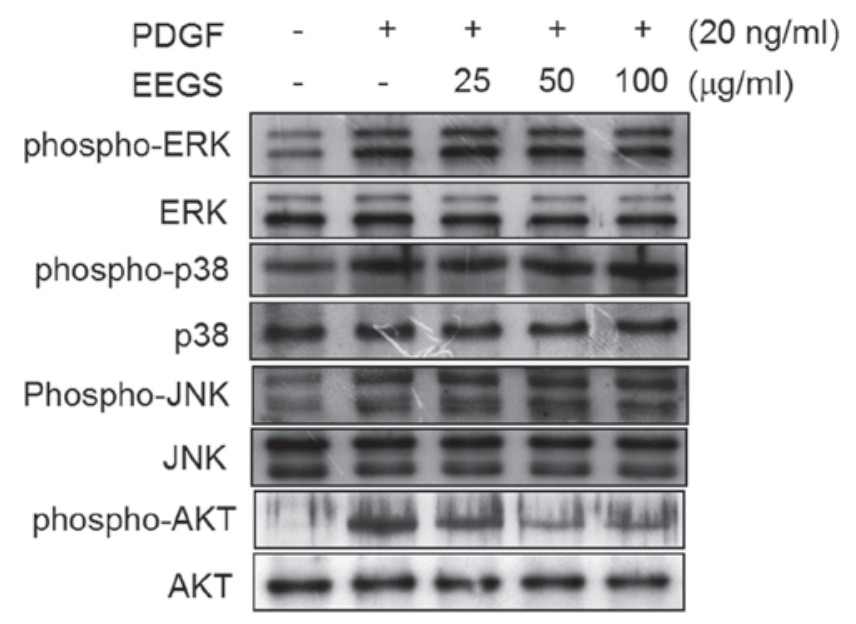

Figure 3. EEGS inhibits PDGF-induced phosphorylation of Akt in VSMCs Quiescent VSMCs were stimulated with PDGF $(20 \mathrm{ng} / \mathrm{ml})$ in the presence or absence of the indicated concentrations of EEGS for $10 \mathrm{~min}$. Western blot analysis was performed using antibodies specific for phospho-ERK1/2, ERK1/2, phospho-p38, p38, phospho-JNK, JNK, phospho-Akt and Akt. PDGF, platelet-derived growth factor; EEGS, ethanol extract of Gleditsia sinensis thorns; VSMC, vascular smooth muscle cell; ERK, extracellular signal-regulated kinase; JNK, c-Jun N-terminal kinase; phospho, phosphorylated.

and p38MAPK was not affected by the addition of EEGS (Fig. 3). These results demonstrate that EEGS may inhibit PDGF-induced VSMC proliferation through the inhibition of Akt phosphorylation.

EEGS prevents the migration of PDGF-induced VSMCs. The migration and invasion of VSMCs are highly associated with the formation of vascular lesions (9). Therefore, wound-healing migration and Matrigel $^{\mathrm{TM}}$ invasion assays were used to investigate the role of EEGS in the regulation of VSMC migration and invasion. Serum-starved cells were wounded and incubated in the presence or absence of PDGF. VSMC wounds were allowed to heal for $24 \mathrm{~h}$ subsequent to the addition of PDGF. As shown in Fig. 4A, PDGF treatment was observed to significantly increase VSMC migration. Furthermore, pretreatment with EEGS was found to markedly inhibit the migration of VSMCs induced by PDGF at $24 \mathrm{~h}$ (Fig. 4A). To confirm the inhibitory effect of EEGS on PDGF-induced migration, a Matrigel invasion assay was performed. PDGF treatment was associated with a marked increase in VSMC invasiveness through the Matrigel-plated Boyden chamber at $24 \mathrm{~h}$, which was reduced with the addition of EEGS (Fig. 4B). These results suggest that EEGS is a potent inhibitor of PDGF-induced migration in VSMCs.

EEGS inhibits PDGF-stimulated MMP-9 expression through the suppression of $N F-\kappa B, A P-1$ and $S p-1$ binding activities. MMP-9 expression is closely associated with VSMC migration and invasion from media to intima (9-13). In order to assess the role of EEGS in the expression of MMP-9, a gelatin zymographic assay was performed. Media from VSMCs induced by PDGF showed proteolytic activity at $92 \mathrm{kDa}$, corresponding to MMP-9 (Fig. 5A). This induction of MMP-9 expression by PDGF was suppressed following EEGS treatment (Fig. 5A). Similar results were observed with western blot analysis (Fig. 5A). Furthermore, under similar experimental conditions, MMP-2 expression was inhibited by the addition of EEGS to PDGF-treated VSMCs (Fig. 5A). To further understand the mechanism underlying this suppressive effect on MMP-9 expression, an EMSA assay was performed using three motifs: 
A

Control

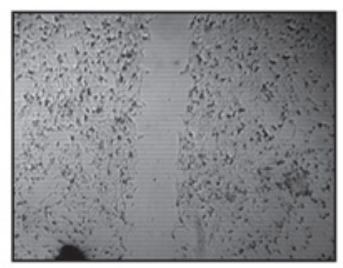

B

$24 \mathrm{~h}$

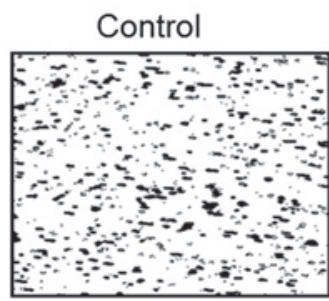

PDGF

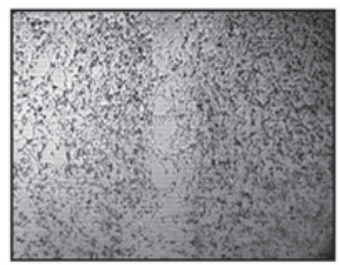

PDGF

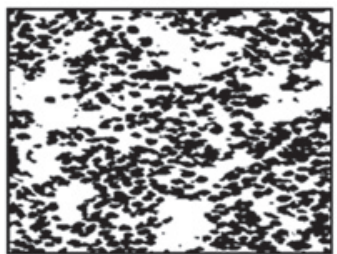

PDGF + EEGS

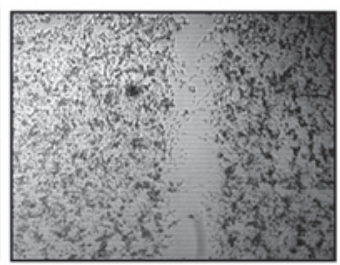

PDGF + EEGS

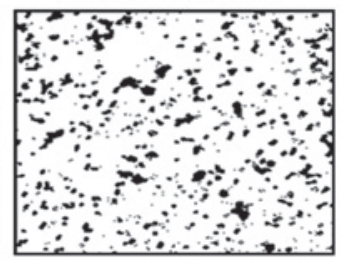

EEGS

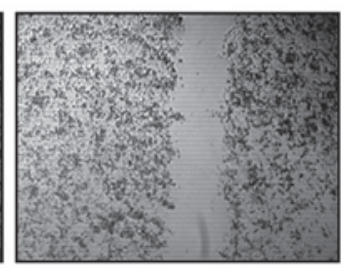

EEGS

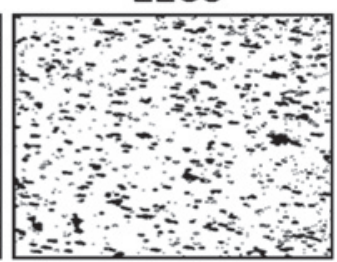

Figure 4. EEGS inhibits the migration and invasion of VSMCs induced by PDGF. (A) Quiescent VSMCs were pretreated with $100 \mu \mathrm{g} / \mathrm{ml}$ EEGS for $40 \mathrm{~min}$, prior to the addition of PDGF (20 ng/ml) for $24 \mathrm{~h}$. Cell wound closure was monitored using phase microscopy. (B) Quiescent VSMCs were cultured in Matrigel $^{\mathrm{TM}}$-coated membranes of Transwell plates and then incubated in $100 \mu \mathrm{g} / \mathrm{ml}$ EEGS for $40 \mathrm{~min}$, prior to stimulation with PDGF (20 ng/ml) for $24 \mathrm{~h}$. Invaded cells were determined by staining cells that had migrated through the pores (magnification, x40). PDGF, platelet-derived growth factor; EEGS, ethanol extract of Gleditsia sinensis thorns; VSMC, vascular smooth muscle cell.

A

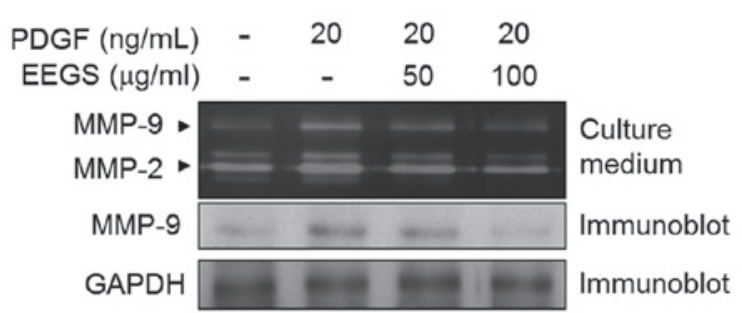

B

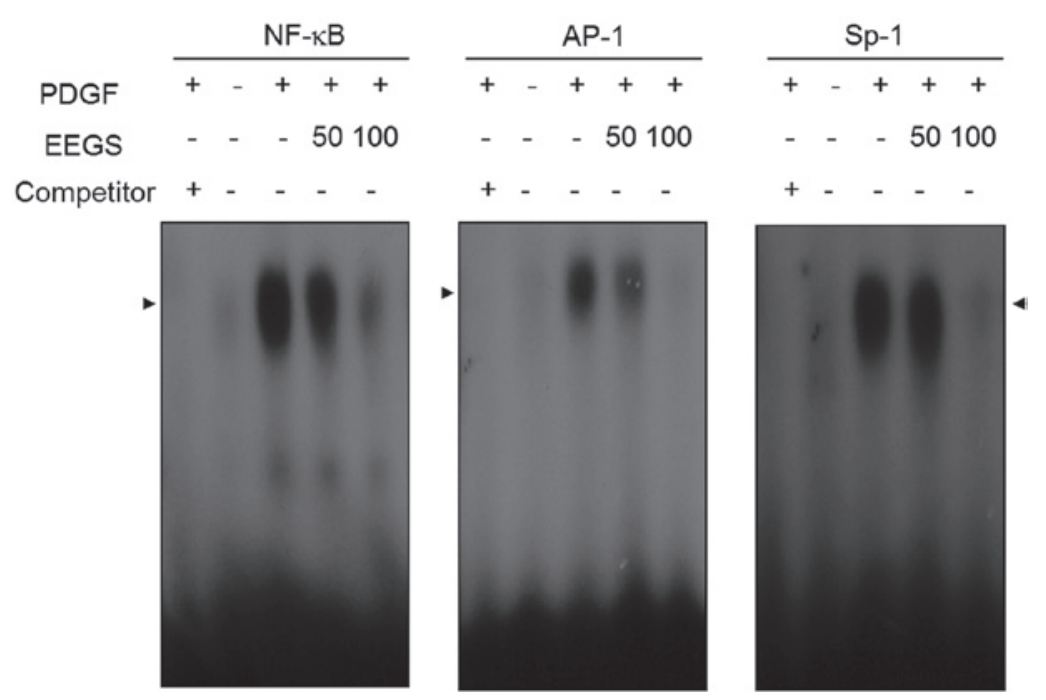

Figure 5. EEGS suppresses MMP-9 expression through the decreased binding activities of NF-кB, AP-1 and Sp-1 motifs in PDGF-treated VSMCs. Quiescent VSMCs were incubated in EEGS $(100 \mu \mathrm{g} / \mathrm{ml})$ for $40 \mathrm{~min}$, prior to stimulation with PDGF $(20 \mathrm{ng} / \mathrm{ml})$ for $24 \mathrm{~h}$. (A) Culture supernatants were examined zymographically for MMP activity. Western blot analysis of cell lysates was performed using antibodies specific for MMP-9. (B) The activated NF- $\mathrm{B}$, AP-1 and Sp-1 from the nuclear extract were analyzed using electrophoretic mobility shift assay with radiolabeled oligonucleotide probes. PDGF, platelet-derived growth factor; EEGS, ethanol extract of Gleditsia sinensis thorns; VSMC, vascular smooth muscle cell; MMP, matrix metalloproteinase; NF-кB; nuclear factor $\kappa$-light-chain-enhancer of activated B cells; AP, activator protein; SP, specificity protein.

NF- $\mathrm{kB}, \mathrm{AP}-1$ and Sp-1 cis-elements. Nuclear extracts from VSMCs treated with PDGF markedly increased the binding activities of NF- $\mathrm{KB}$, AP-1 and Sp-1 (Fig. 5B). Furthermore, EEGS treatment was observed to inhibit the increased binding activity of the NF-кB, AP-1 and Sp-1 motifs (Fig. 5B). These data demonstrate that EEGS treatment is capable of inhibiting MMP-9 expression, at least in part, by inhibiting the binding activities of the NF-kB, AP-1 and Sp-1 transcription factors. 


\section{Discussion}

The thorns of Gleditsia sinensis have demonstrated pharmacological effects on several systems, including anti-cancer, anti-microbial and anti-inflammatory effects $(17,20,21)$. A previous study revealed the inhibitory effect of EEGS on the proliferation of VSMCs (23). However, the molecular mechanism of EEGS on PDGF-stimulated VSMC responses is yet to be elucidated. The present study aimed to investigate the precise mechanism of the anti-proliferative effect of EEGS in PDGF-induced VSMCs. PDGF-induced VSMC proliferation has a significant role in the pathogenesis of vascular lesion formation $(4,5)$.

Under normal conditions, VSMCs maintain a quiescent status. However, subsequent to vascular injury, such as growth factor stimulation, VSMCs re-enter the cell cycle in a state of abnormal proliferation (1). It is well established that PDGF is a key growth factor involved in VSMC phenotypic change $(4,5)$ In accordance with this, the present study showed that PDGF induced the proliferation of VSMCs through cell-cycle progression. Thus, in the present study, it was hypothesized that EEGS was likely to inhibit cell proliferation through the induction of cell-cycle arrest in PDGF-stimulated VSMCs. As revealed by the MTT and thymidine uptake assays, EEGS inhibited the PDGF-induced proliferation of VSMCs without cytotoxicity in vitro. The inhibitory effect of EEGS was associated with the accumulation of cells in the $G_{1}$-phase of the cell cycle. To further elucidate the effect of EEGS on cell-cycle control, the role of cyclin-CDK complexes, which induce a complex cascade of events (6-8) during the induction of the $\mathrm{G}_{1}$-phase cell-cycle arrest, was examined in the PDGF-stimulated VSMCs treated with EEGS. The expression of cyclins and CDKs, cell-cycle regulatory proteins that are essential for $\mathrm{G}_{1}$ - to $\mathrm{S}$-phase progression, was investigated (6-8). The results of the present study showed that EEGS treatment significantly downregulated cyclin D1, cyclin E, CDK2 and CDK4 expression in PDGF-treated VSMCs. In addition, the kinase activities associated with CDK2 and 4 were inhibited by EEGS in PDGF-treated VSMCs. These results demonstrate that the anti-proliferative effect of EEGS may be caused by the $\mathrm{G}_{1}$-phase cell-cycle arrest associated with the downregulation of cyclins and CDKs in PDGF-stimulated VSMCs.

The activity of cyclin/CDK complexes is highly controlled by the binding of the CDKIs p21WAF1 and p27KIP1 $(7,8)$. CDKIs bind tightly to the cyclin/CDK complexes and inhibit their activity, resulting in an accumulation of cells at the $\mathrm{G}_{1}$-phase boundary $(7,8)$. It has been previously reported that p27KIP1 expression is downregulated following vascular injury (24). Furthermore, it has been shown that overexpression of p27KIP1 has a suppressive effect on intimal VSMCs, reducing neointimal hyperplasia in rat carotid arteries (25). The present study showed an inhibition of p27KIP1 expression in PDGF-treated VSMCs. This effect was reversed following EEGS treatment, suggesting that the EEGS-induced accumulation of p27KIP1 could be responsible for the $G_{1}$-phase arrest observed in the PDGF-treated VSMCs. p21WAF1 was originally described as an inhibitor of cell proliferation $(7,8)$. However, several studies have demonstrated that p21WAF1 is involved in VSMC proliferation $(26,27)$. In the present study, PDGF was found to induce the expression of p21WAF1, and this effect was not changed in VSMCs following EEGS treatment. These results demonstrate that the $\mathrm{G}_{1}$-phase cell-cycle arrest induced by EEGS is due to the decreased expression of cyclin/CDK complexes through the induction of p27KIP1 in PDGF-treated VSMCs.

MAPK and Akt are closely implicated in VSMC proliferation induced by mitogenic stimuli, such as PDGF $(4,5)$. Therefore, the effect of the early signal transduction pathway in response to PDGF stimulation in VSMCs was examined. PDGF treatment has previously been demonstrated to induce the phosphorylation of Akt and MAPKs, including ERK1/2, JNK and p38MAPK, in VSMCs $(4,5)$. In accordance with these previous findings, EEGS treatment in the present study was found to significantly attenuate PDGF-induced Akt phosphorylation in VSMCs. However, EEGS treatment was not observed to affect the phosphorylation of MAPKs, including ERK1/2, JNK and p38MAPK, in PDGF-treated VSMCs. These results suggest that EEGS inhibited PDGF-stimulated proliferation in VSMCs through the suppression of Akt phosphorylation. Although a previous study has reported that the phosphorylation of MAPKs, including ERK1/2, JNK and p38MAPK, is induced following EEGS treatment in colon cancer cells (21), to the best of our knowledge, this is the first study to show that the suppression of Akt phosphorylation is involved in the EEGS-induced inhibition of cell proliferation.

It is well established that the migration and invasion of VSMCs have a role in atherosclerotic lesion formation $(1,3,9)$. Several studies have demonstrated that cytokines and growth factors, produced by various types of stimuli, promote the invasion and migration of VSMCs $(1,3,9)$. The enhanced invasive and migratory capacity of VSMCs has been associated with the presence of the growth factor PDGF $(1,9)$. In the present study, a wound-healing assay revealed an upregulation in migratory potential in VSMCs treated with PDGF. Similar results were observed using a Matrigel invasion assay. Of note, the significant reduction in the migratory and invasive capacity observed in PDGF-stimulated VSMCs treated with EEGS was not a consequence of cell viability. These results demonstrate that EEGS may be an inhibitor of the migration and invasion that is induced by PDGF in VSMCs.

VSMC migration requires degradation of the ECM, which results in the formation of neointimal lesions. The primary mechanism involved in VSMC migration is the production of proteolytic enzymes, including MMP-2 and -9 (9-13). A growing body of evidence suggests that MMP-9 expression may contribute to the enhanced progression of arterial vascular lesions (9-13). Therefore, in the present study, MMP-9 expression was assessed in PDGF-treated VSMCs. In accordance with the present migration and invasion results, MMP-9 expression was increased with PDGF treatment. To further elucidate the regulation of MMP-9 expression by PDGF in VSMCs, an EMSA assay was performed. In the present study, the transcription

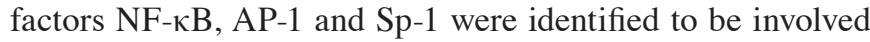
in MMP-9 expression in PDGF-stimulated VSMCs. Therefore, NF- $\kappa$ B, AP-1 and Sp-1 may co-operate in the activation of the MMP-9 gene in PDGF-treated VSMCs. Next, the effect of EEGS treatment on the inhibition of MMP-9 expression was examined in PDGF-induced VSMCs. EEGS was observed to reduce PDGF-stimulated MMP-9 expression in VSMCs, as determined by zymography and western blot analyses. 
Furthermore, an EMSA assay using consensus NF-кB, AP-1 and Sp-1 probes showed that EEGS treatment induced a significant decrease in the binding activities of NF-kB, AP-1 and Sp-1 in PDGF-treated VSMCs. These results showed that the ability of EEGS to inhibit MMP-9 expression in PDGF-treated VSMCs may be achieved through the suppression of NF- $\mathrm{kB}, \mathrm{AP}-1$ and Sp-1 binding activities.

The accumulation of VSMCs in the arterial intima is a key event in the pathogenesis of cardiovascular diseases, which is characterized by the formation of neointima resulting from the proliferation and migration of VSMCs from media to intima (1). A proliferating and migrating cell population and increased PDGF expression are observed in the neointimal layer of severely injured arteries (28), suggesting that the PDGF-induced proliferation and migration of VSMCs are important features in neointimal formation following vascular injury, and may lead to cardiovascular disease $(4,5,28)$. Results from the present study demonstrated that EEGS treatment significantly inhibited proliferation and migration in PDGF-stimulated VSMCs, without cell toxicity. The proliferation and migration of VSMCs is a key factor in neointimal formation; therefore, the results of this study suggest that an additional mechanism exists by which EEGS treatment may be critical in preventing the progression of atherosclerosis and restenosis.

In conclusion, to the best of our knowledge, the present study has provided the first evidence that EEGS inhibits the proliferation of PDGF-simulated VSMCs via Akt phosphorylation without cell death. EEGS also induced $\mathrm{G}_{1}$-phase cell-cycle arrest, as a result of the decreased levels of cyclin D1/CDK4 and cyclin E/CDK2 that were mediated by the upregulation of p27KIP1 expression. In addition, EEGS treatment was found to suppress migration and invasion in PDGF-stimulated VSMCs. Furthermore, EEGS markedly reduced the PDGF-induced expression of MMP-9 through the suppression of NF- $\kappa \mathrm{B}, \mathrm{AP}-1$ and Sp-1 binding activities. The results of the present study may, in part, explain the therapeutic potential of EEGS for the prevention of cardiovascular diseases associated with multiple pathological events involving the proliferation and migration of VSMCs.

\section{Acknowledgements}

This study was supported by the Basic Science Research Program through the National Research Foundation of Korea (NRF), funded by the Ministry of Education Science and Technology (grant no. 2008-0062611). This study was also supported by the Chung-Ang University research grants 2013.

\section{References}

1. Ross R: Cell biology of atherosclerosis. Annu Rev Physiol 57: 791-804, 1995

2. Dzau VJ, Braun-Dullaeus RC and Sedding DG: Vascular proliferation and atherosclerosis: new perspectives and therapeutic strategies. Nat Med 8: 1249-1256, 2002.

3. Doran AC, Meller N and McNamara CA: Role of smooth muscle cells in the initiation and early progression of atherosclerosis. Arterioscler Thromb Vasc Biol 28: 812-819, 2008.

4. Heldin $\mathrm{CH}$ and Westermark B: Mechanism of action and in vivo role of platelet-derived growth factor. Physiol Rev 79: 1283-1316, 1999.
5. Zhan Y, Kim S, Izumi Y, Izumiya Y, Nakao T, Miyazaki H and Iwao H: Role of JNK, p38, and ERK in platelet-derived growth factor-induced vascular proliferation, migration, and gene expression. Arterioscler Thromb Vasc Biol 23: 795-801, 2003.

6. Sherr CJ: G1 phase progression: cycling on cue. Cell 79: 551-555, 1994.

7. Xiong Y, Hannon GJ, Zhang H, Casso D, Kobayashi R and Beach D: p21 is a universal inhibitor of cyclin kinases. Nature 366: 701-704, 1993.

8. Toyoshima $\mathrm{H}$ and Hunter T: p27, a novel inhibitor of G1 cyclin-cdk protein kinase activity, is related to p21. Cell 78: 67-74, 1994.

9. Newby AC and Zaltsman AB: Molecular mechanisms in intimal hyperplasia. J Pathol 190: 300-309, 2000.

10. Cho A and Reidy MA: Matrix metalloproteinase-9 is necessary for the regulation of smooth muscle cell replication and migration after arterial injury. Circ Res 91: 845-851, 2002.

11. Galis ZS, Johnson C, Godin D, Magid R, Shipley JM, Senior RM and Ivan E: Targeted disruption of the matrix metalloproteinase-9 gene impairs smooth muscle cell migration and geometrical arterial remodeling. Circ Res 91: 852-859, 2002.

12. Cho A, Graves J and Reidy MA: Mitogen-activated protein kinases mediate matrix metalloproteinase-9 expression in vascular smooth muscle cells. Arterioscler Thromb Vasc Biol 20: 2527-2532, 2000.

13. Moon SK, Cha BY and Kim CH: ERK1/2 mediates TNF-alpha-induced matrix metalloproteinase-9 expression in human vascular smooth muscle cells via the regulation of NF-kappaB and AP-1: Involvement of the ras dependent pathway. J Cell Physiol 198: 417-427, 2004.

14. Dollery CM, McEwan JR and Henney AM: Matrix metalloproteinases and cardiovascular disease. Circ Res 77: 863-868, 1995.

15. Hansson GK and Robertson AK: TGF-beta in atherosclerosis. Arterioscler Thromb Vasc Biol 24: E137, 2004.

16. Lim JC, Park JH, Budesinsky M, Kasal A, Han YH, Koo BS, Lee SI and Lee DU: Antimutagenic constituents from the thorns of Gleditsia sinensis. Chem Pharm Bull (Tokyo) 53: 561-564, 2005.

17. Zhou L, Li D, Wang J, Liu Y and Wu J: Antibacterial phenolic compounds from the spines of Gleditsia sinensis Lam. Nat Prod Res 21: 283-291, 2007.

18. Li WH, Zhang XM, Tian RR, Zheng YT, Zhao WM and Qiu MH: A new anti-HIV lupane acid from Gleditsia sinensis Lam. J Asian Nat Prod Res 9: 551-555, 2007.

19. Shin TY and Kim DK: Inhibitory effect of mast cell-dependent anaphylaxis by Gleditsia sinensis. Arch Pharm Res 23: 401-406, 2000.

20. Park E and Shin MJ: Anti-inflammatory activity of aqueous extract from Gleditsiae Spina. J Pharmaceut Soc Korea 37: 124-128, 1993.

21. Lee SJ, Cho YH, Kim H, Park K, Park SK, Ha SD, Kim WJ and Moon SK: Inhibitory effects of the ethanol extract of Gleditsia sinensis thorns on human colon cancer HCT116 cells in vitro and in vivo. Oncol Rep 22: 1505-1512, 2009.

22. Zhou L, Li D, Jiang W, Qin Z, Zhao S, Qiu M and Wu J: Two ellagic acid glycosides from Gleditsia sinensis Lam. with antifungal activity on Magnaporthe grisea. Nat Prod Res 21: 303-309, 2007.

23. Lee SJ, Park SS, Kim WJ and Moon SK: Gleditsia sinensis thorn extract inhibits proliferation and TNF- $\alpha$-induced MMP-9 expression in vascular smooth muscle cells. Am J Chin Med 40: 373-386, 2012.

24. Tanner FC, Yang ZY, Duckers E, Gordon D, Nabel GJ and Nabel EG: Expression of cyclin-dependent kinase inhibitors in vascular disease. Circ Res 82: 396-403, 1998.

25. Chen D, Krasinski K, Sylvester A, Chen J, Nisen PD and Andrés V: Downregulation of cyclin-dependent kinase 2 activity and cyclin A promoter activity in vascular smooth muscle cells by p27(KIP1), an inhibitor of neointima formation in the rat carotid artery. J Clin Invest 99: 2334-2341, 1997.

26. Moon SK, Kim HM, Lee YC and Kim CH: Disialoganglioside (GD3) synthase gene expression suppresses vascular smooth muscle cell responses via the inhibition of ERK1/2 phosphorylation, cell cycle progression, and matrix metalloproteinase-9 expression. J Biol Chem 279: 33063-33070, 2004.

27. Shankland SJ and Wolf G: Cell cycle regulatory proteins in renal disease: role in hypertrophy, proliferation, and apoptosis. Am J Physiol Renal Physiol 278: F515-F529, 2000.

28. Uchida K, Sasahara M, Morigami N, Hazama F and Kinoshita M: Expression of platelet-derived growth factor B-chain in neointimal smooth muscle cells of balloon injured rabbit femoral arteries. Atherosclerosis 124: 9-23, 1996. 